\title{
Germinación de semillas de ají (Capsicum sinense) estimuladas electromagnéticamente
}

\section{Electromagnetic stimulated chili (Capsicum sinense) seed germination}

CRISTIAN JIMÉNEZ1

MELISSA MÉNDEZ2

MARTHA CONSTANZA DAZA ${ }^{2,3}$

ORLANDO ZÚÑIGA'

Germinación de semillas

de ají estimuladas

electromagnéticamente.

Foto: C. Jiménez

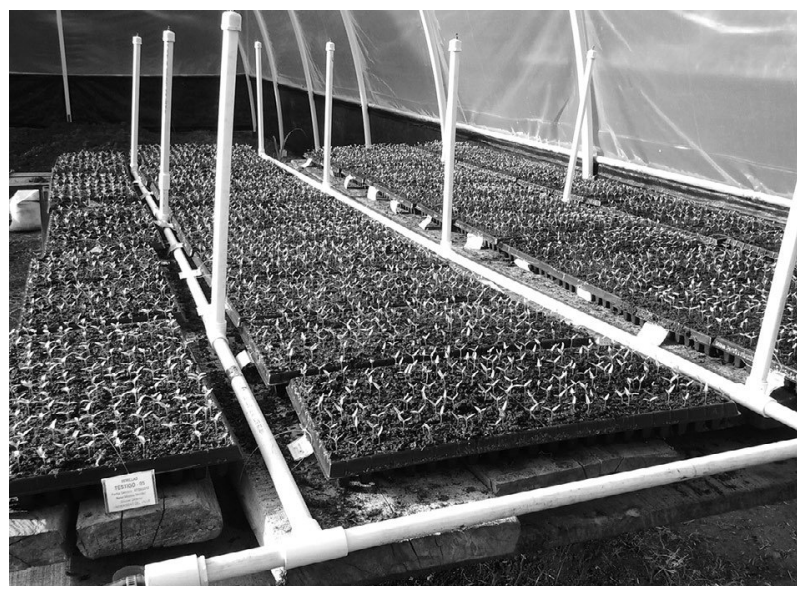

\section{RESUMEN}

Los campos magnéticos tienen efecto sobre los sistemas vivientes y materiales biológicos, especialmente en el incremento de la germinación de semillas y el aumento de biomasa en plantas. Con el objetivo de determinar el efecto de los campos electromagnéticos sobre el tiempo y el porcentaje de germinación de semillas de ají, var. Limo Rojo, se utilizaron bobinas para generar campos electromagnéticos continuos (DC) y alternos (AC) a intensidades de 30 y 60 militeslas (mT) y tiempos de exposición de las semillas de 30, 60 y 120 min, con un diseño factorial de múltiples niveles completamente al azar. Se encontraron diferencias significativas entre los tratamientos en la cantidad de semillas germinadas por día, siendo más marcado a partir del séptimo día después de la siembra hasta el doceavo día, mostrando que al incrementar la intensidad y el tiempo de exposición al campo electromagnético, se redujo el tiempo de germinación. En lo que respecta al porcentaje de germinación final, no se encontraron diferencias significativas entre tratamientos. Los campos electromagnéticos tuvieron efecto en el tiempo de germinación mas no en el porcentaje de germinación de semillas de ají.

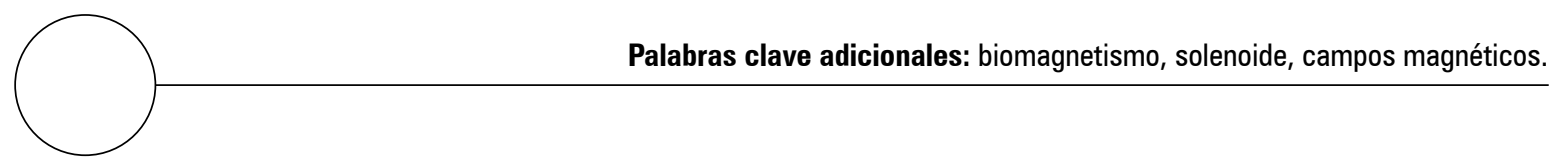

Facultad de Ciencias, Departamento de Física, Universidad del Valle, Cali (Colombia).

Escuela de Ingeniería de Recursos Naturales y del Ambiente (Eidenar), Universidad del Valle, Cali (Colombia).

Autor para correspondencia: martha.daza@correounivalle.edu.co 


\section{ABSTRACT}

Magnetic fields have effects on living systems and biological materials, particularly in seed germination and plant biomass increment. In order to determine the effect of electromagnetic fields on time and percentage of chili var. Limo Rojo seed germination, coils were used to generate continuous (DC) and alternating (AC) electromagnetic fields with intensities of 30 and $60 \mathrm{mT}$, and exposure times of seeds of 30,60 and 120 min, with a completely random multi-level factorial design. Significant differences were found between the treatments for the number of germinated seeds per day, especially on the seventh day after planting until the twelfth day, where the germination time was reduced with intensity and time of exposure to the electromagnetic field increment. For the percentage of germination, no significant differences were found between the treatments. The electromagnetic fields had an effect on germination time but not on percentage of chili germination.

Additional key words: biomagnetism, solenoid, magnetic fields.

El estudio de los efectos de los campos magnéticos sobre el crecimiento de plantas es un tema desarrollado desde hace varios años. Entre los primeros trabajos se encuentra el realizado por Albert Roy Davies, quien logró una patente en 1950 por tratar las semillas magnéticamente y conseguir así estimular su crecimiento (Biomagnetics USA, 2013).

En los últimos años las investigaciones enfocadas a determinar el efecto de los campos magnéticos sobre diferentes semillas ha ido en aumento; el objetivo de estas investigaciones se ha centrado en identificar las características de los campos magnéticos como son la intensidad (magnitud del campo), frecuencia (número de ciclos por unidad de tiempo) y el tiempo de estimulación necesarias para mejorar el desarrollo y rendimiento de plantas.

Entre las investigaciones realizadas se han analizado plantas de tomate (De Souza et al., 1999), fríjol (Vásquez et al., 2006), maíz (Racuciu et al., 2008), girasol (Vashisth y Nagarajan, 2010), papa (Marks y Szecówka, 2010) entre otras especies, obteniendo resultados positivos con tratamientos magnéticos (campos generados por imanes) y electromagnéticos (campos generados por corrientes eléctricas) en la germinación y el crecimiento de dichas plantas. Estudios como los de Vashisth y Nagarajan (2010) han mostrado que estos tratamientos tienen incidencia en el aumento de la actividad de la $\alpha$-amilasa, deshidrogenasa y las actividades proteicas (degradación enzimática de proteínas o actividad catalítica) de las semillas expuestas magnéticamente, que estimulan la rápida germinación y mejor desarrollo de plantas. Estos resultados conducen a que la técnica de estimulación magnética de semillas tenga un potencial para su aplicación en diferentes cultivos, en especial los de mayor interés productivo.

En Colombia, el ají (Capsicum sinense Jacques) se ha convertido en una de las más importantes hortalizas de exportación. Según el Ministerio de 
Agricultura y Desarrollo Rural (2012), durante el 2011 se sembraron en Colombia 2.045 ha de ají, con una producción total de 16.515 t y un rendimiento promedio de $8.078 \mathrm{~kg} \mathrm{ha}^{-1}$, de las cuales el $95 \%$ de la producción es para exportación con destino a Estados Unidos. La proyección a 10 años es tener cultivadas 1.000 ha en el Valle del Cauca y producir $25.000 \mathrm{t}$ de ají fresco por año, pues la demanda mundial es creciente y los grandes productores tienen los ojos puestos en países tropicales como Colombia (Revista Cambio, 2008).

Para incrementar la producción de ají se requiere semilla de calidad que permita lograr un buen establecimiento y desarrollo del cultivo a través de tecnologías económicas, de fácil acceso y amigables con el ambiente. En este sentido, la estimulación electromagnética puede ser una tecnología interesante para el acondicionamiento de semillas que, en diversos cultivos, ha dado excelentes resultados, pero que en el caso particular del ají aún no se ha evaluado de forma suficiente en todas las variedades.

El presente trabajo tuvo como objetivo estudiar el uso de la aplicación de campos electromagnéticos en semillas de ají, variedad Limo Rojo, eva- luando el tiempo y porcentaje de germinación de las semillas, como estrategia sostenible para el manejo eficiente del cultivo.

\section{MATERIALES Y MÉTODOS}

El ensayo se realizó bajo condiciones de invernadero en la granja experimental de la Universidad del Valle, Cali, la cual se encuentra a una altitud de 995 msnm y ubicada a 322'33,55” N y $76^{\circ} 31^{\prime} 58,43^{\prime \prime} \mathrm{W}$; cuenta con una temperatura promedio anual de $23^{\circ} \mathrm{C}$, humedad relativa del $73 \%$, precipitación promedio anual de $908 \mathrm{~mm}$ y $162 \mathrm{~h}$ en promedio de brillo solar.

\section{Equipo de estimulación electromagnética}

La adecuación del equipo de estimulación electromagnética consistió en el montaje del circuito para generar campos electromagnéticos de dos tipos: continuos y alternos.

En la figura 1 se detallan las partes básicas del circuito para generar campos electromagnéticos, las cuales constan de un solenoide o bobina, un amperímetro para medir la corriente y una fuen-

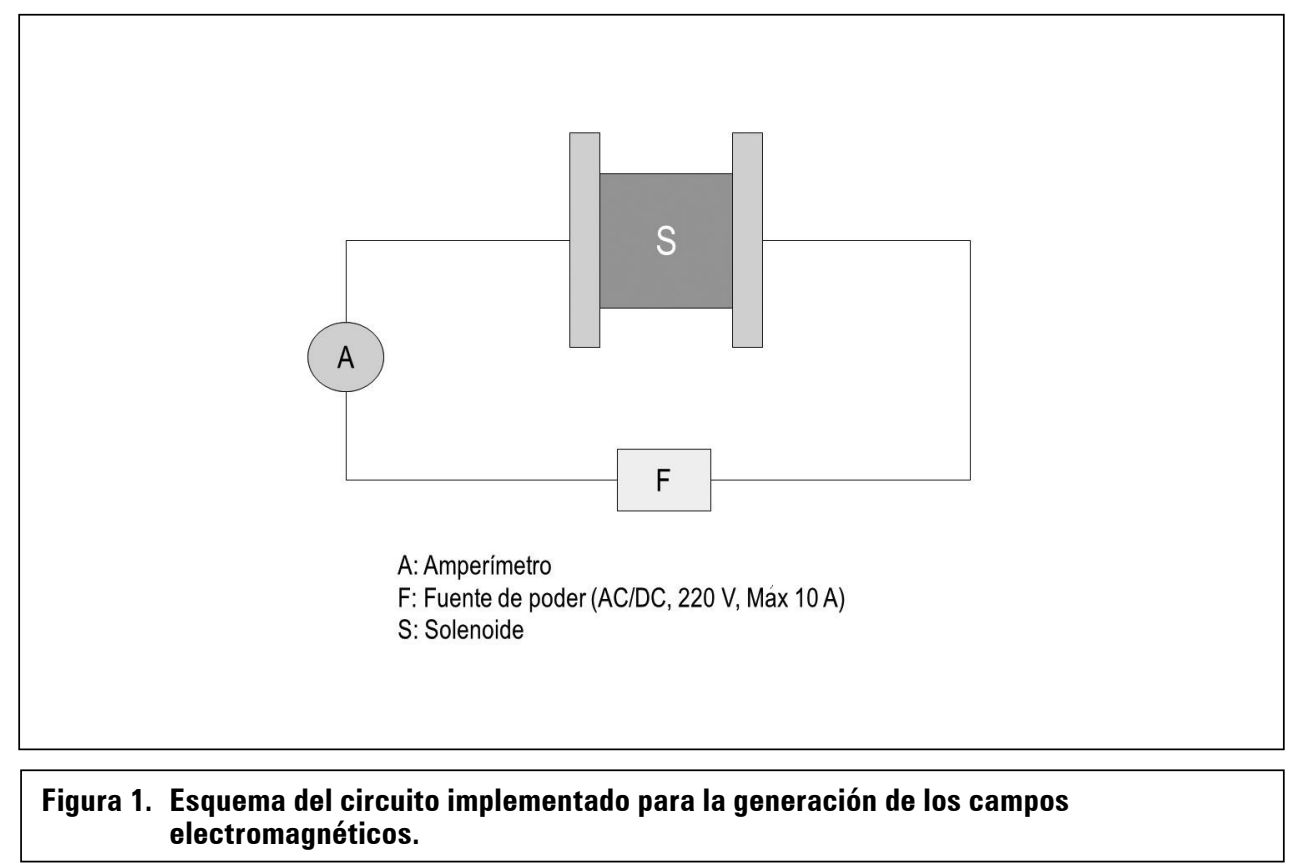

Rev. Colomb. Cienc. Hortic. 
te de poder conectados en serie, además de un sistema de refrigeración con agua para regular la temperatura durante el proceso de estimulación.

Las bobinas utilizadas para la estimulación electromagnética constaban de 20 capas y fueron diseñadas y construidas con alambre número 14, con 57 espiras por capa. La longitud de la bobina fue de 0,100 m y el diámetro de su núcleo de 0,076 m (figura 2). Después de la construcción se realizó la curva de calibración de los solenoides con el fin de conocer la relación entre campo electromagnético generado (B) y corriente eléctrica (I); para ello se utilizó un teslámetro marca Phywe (Phywe Systeme GmbH, Göttingen, Alemania), con su respectiva sonda ubicada en la parte central dentro del solenoide.

\section{Diseño experimental}

El ensayo se ajustó a un diseño factorial de múltiples niveles, dispuestos completamente al azar, con tres factores (tipo de corriente, intensidad de campo y tiempo de exposición). Para el factor tipo de corriente se emplearon dos niveles, corriente continua o directa (DC) y corriente alterna (AC) a $60 \mathrm{~Hz}$, para el factor intensidad de campo se utilizaron dos niveles, 30 y $60 \mathrm{mT}$ y para el factor tiempo de exposición se manejaron tres niveles, 30, 60 y $120 \mathrm{~min}$. Se contó con un tratamiento testigo al cual no se le realizó ningún proceso de estimulación electromagnética. Se tuvo en total 13 tratamientos como lo muestra la tabla 1.

Las unidades experimentales consistieron en bandejas de germinación para 100 plántulas. E1 número de réplicas por tratamiento fue cuatro, para un total de 52 unidades experimentales. Para el desarrollo del montaje experimental, se preseleccionaron 400 semillas de ají (Capsicum sinense Jacques) variedad Limo Rojo provenientes del sur del Valle del Cauca para cada trata-

\section{Tabla 1. Tratamientos implementados para la estimulación electromagnética de semillas de ají.}

\begin{tabular}{|c|l|}
\hline No. & Tratamiento \\
\hline 1 & AC $30 \mathrm{mT} 30^{\prime}$ \\
\hline 2 & AC $30 \mathrm{mT} 60^{\prime}$ \\
\hline 3 & AC $30 \mathrm{mT} 120^{\prime}$ \\
\hline 4 & AC $60 \mathrm{mT} 30^{\prime}$ \\
\hline 5 & AC $60 \mathrm{mT} 60^{\prime}$ \\
\hline 6 & AC $60 \mathrm{mT} 120^{\prime}$ \\
\hline 7 & DC $30 \mathrm{mT} 30^{\prime}$ \\
\hline 8 & DC $30 \mathrm{mT} 60^{\prime}$ \\
\hline 9 & DC $30 \mathrm{mT} 120^{\prime}$ \\
\hline 10 & DC $60 \mathrm{mT} 30^{\prime}$ \\
\hline 11 & DC $60 \mathrm{mT} 60^{\prime}$ \\
\hline 12 & DC $60 \mathrm{mT} 120^{\prime}$ \\
\hline 13 & Testigo \\
\hline
\end{tabular}

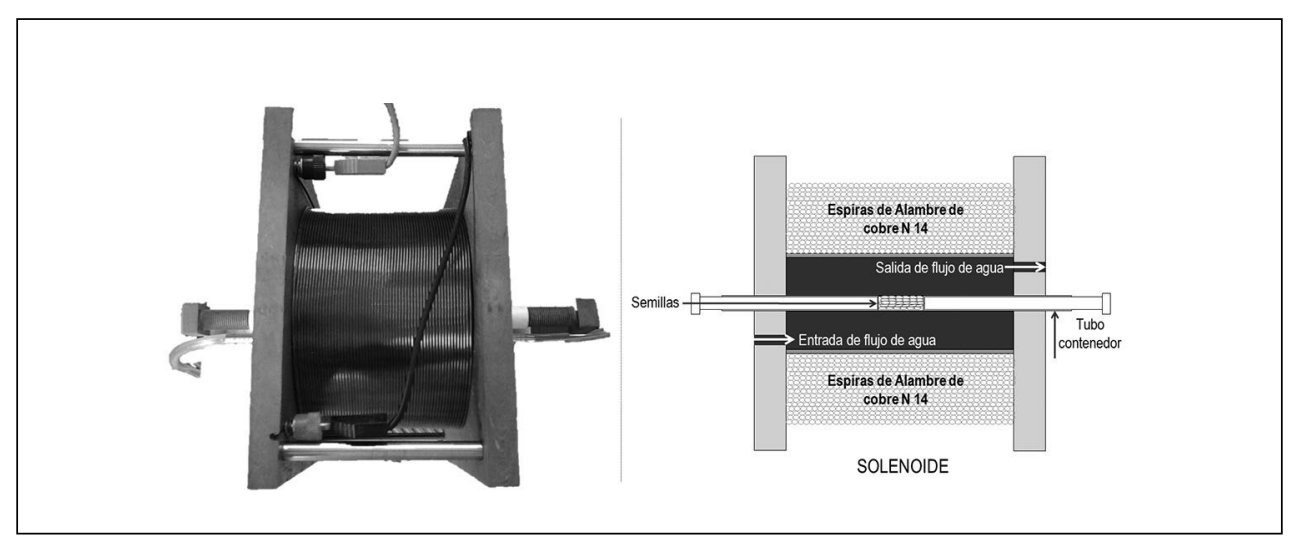

Figura 2. Solenoide implementado para la estimulación electromagnética (izq.) y esquema de la sección transversal del solenoide (der.). 
miento, con el fin de descartar semillas atípicas o deterioradas.

Una vez realizada la preselección de semillas se procedió a su estimulación electromagnética, la cual consistió en ubicarlas en el eje central del solenoide, dentro de un tubo contenedor de PVC de 0,0127 m de diámetro, rodeado por agua en circulación como sistema refrigerante dentro del núcleo, para luego exponerlas a los diferentes tipos de corriente, intensidades y tiempos determinados. Después de la aplicación de los campos electromagnéticos, se realizó la siembra en las bandejas de germinación debidamente rotuladas utilizando turba rubia europea Pindstrup Plus Orange como sustrato; en cada espacio de la bandeja se ubicó una semilla a $1 \mathrm{~cm}$ de profundidad y se cubrió con el sustrato; se llevó cada bandeja al invernadero en donde se realizaron dos riegos por día con un sistema de nebulización, aplicando una lámina de $20 \mathrm{~mm}$ día ${ }^{-1}$.

Las variables de respuesta evaluadas fueron porcentaje de germinación en el tiempo y porcentaje de germinación final. Para su determinación a partir del día 7 se empezó a llevar el correspondiente registro diario del número de plántulas germinadas, hasta el día 19 donde se detuvo este proceso.

\section{Análisis estadístico}

Se realizó un análisis de varianza a los datos obtenidos con un nivel de confianza del 95\% usando el paquete estadístico $R$ de libre distribución. La comparación entre medias se hizo utilizando el test de Tukey con el mismo nivel de confianza.

\section{RESULTADOS Y DISCUSIÓN}

Las figuras 3 y 4 muestran las características de germinación de las semillas de ají en función del tiempo, para los distintos tratamientos.

La germinación de plántulas de ají se inició a partir del día 7 para la mayoría de los tratamientos presentando diferencias significativas hasta el día 12. En este intervalo de tiempo los tratamientos AC-60mT-120' y DC-60mT-120'

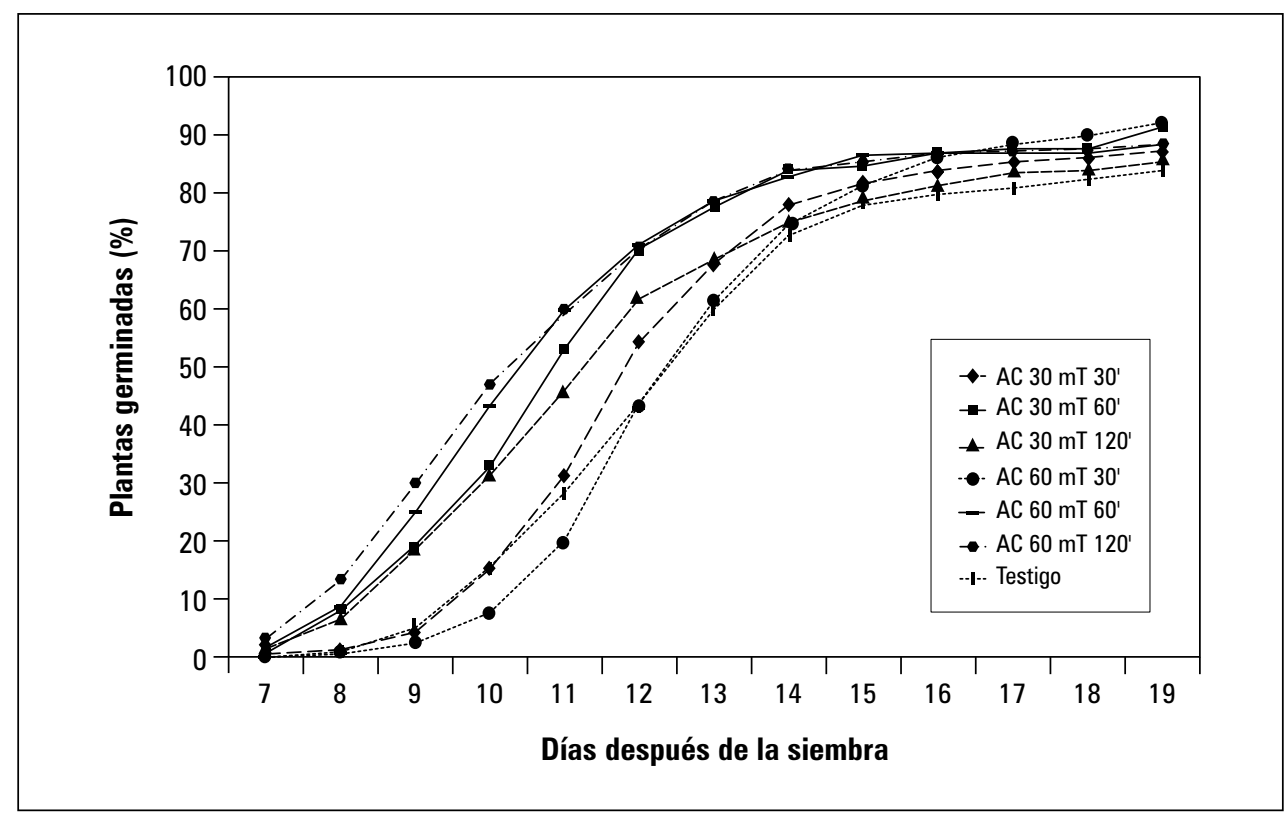

Figura 3. Porcentaje de germinación de semillas de ají en el tiempo para los tratamientos con campo alterno (AC) y tratamiento testigo. 


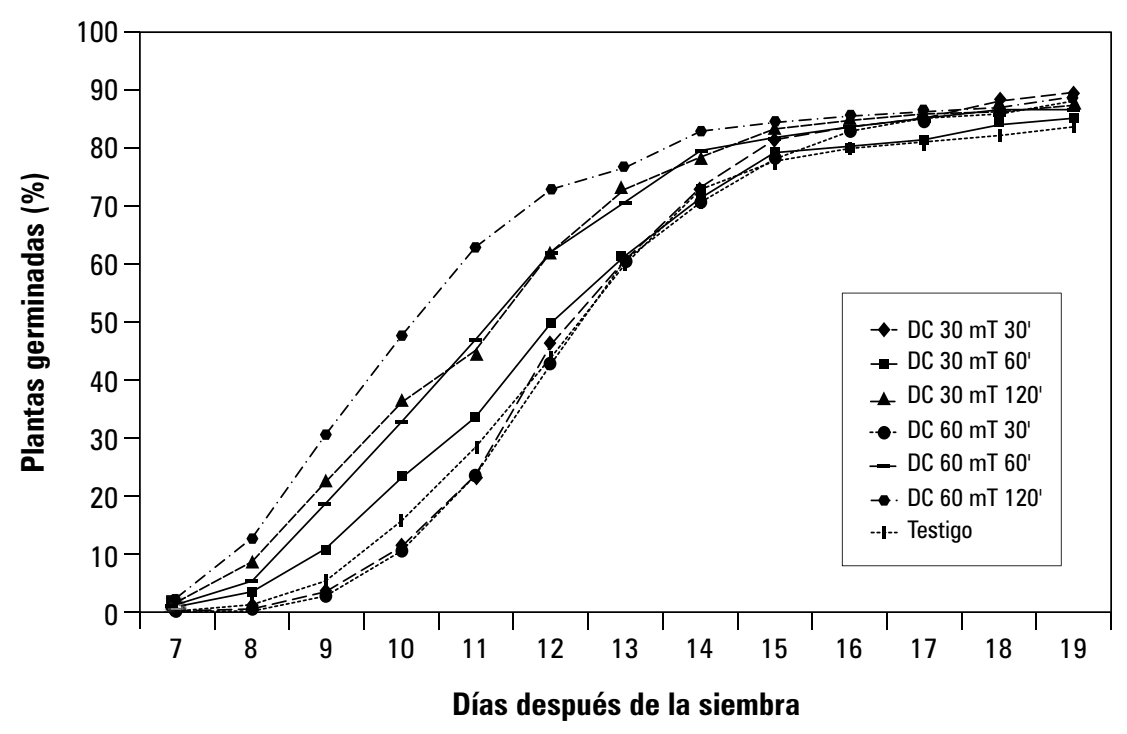

Figura 4. Porcentaje de germinación de semillas de ají en el tiempo para los tratamientos con campo continuo (DC) y tratamiento testigo.

fueron los que obtuvieron el mayor número de semillas germinadas mientras que los tratamientos DC-30mT-30', AC-60mT-30' y DC$60 \mathrm{mT}-30$ ' fueron los que obtuvieron el menor valor de semillas. No se encontraron diferencias significativas entre tipos de corriente pero sí entre intensidades del campo magnético, con tendencia a presentar mejor porcentaje de germinación con el campo de $60 \mathrm{mT}$. En cuanto al tiempo de exposición al campo electromagnético se encontró que a mayor tiempo, mayor fue el porcentaje de germinación. Al compararlos con el testigo, el tratamiento AC-60mT-120' aumentó el número de plántulas germinadas en un $100 \%, 93 \%, 83 \%, 66 \%, 52 \%$ y $38 \%$ para los días 7, 8, 9, 10, 11 y 12 respectivamente. A partir del día 13 los valores de germinación diarios fueron similares, sin diferencias significativas entre tratamientos (tabla 2).

Los factores tiempo e intensidad de campo fueron significativos en los días 7, 8, 9, 10, 11 y 12 en el proceso de germinación de las semillas de ají. La interacción de los factores tiempo e intensidad de campo también resultó ser significativa, al igual que la interacción entre los factores tipo de campo y tiempo. Estos resultados concuerdan con los estudios obtenidos por Marks y Szecówka (2010), quienes encontraron que la exposición tuvo un efecto significativamente positivo y directamente proporcional a la inducción magnética y al tiempo empleado, sobre la germinación con la estimulación de semillas de papa (Solanum tuberosum) con campo magnético variable. También Torres et al. (2008) encontraron que el tiempo de exposición fue el factor de mayor incidencia en la germinación de las semillas de arroz (Oryza sativa) y tomate (Solanum lycopersicum).

Los resultados indicaron que al incrementar la intensidad y el tiempo de exposición al campo electromagnético, se redujo el tiempo de germinación de las semillas de ají 'Limo Rojo'. El efecto de la aplicación de campos magnéticos en la germinación de las semillas no está muy bien identificado (Flórez et al., 2007) ; sin embargo, su respuesta se atribuye a diversos mecanismos como estimulación enzimática de la $\alpha$-amilasa y $\beta$-amilasa(Rochalska y Grabowska, 2007; Vashisth y Nagarajan, 2010), la dinámica de absor- 
Tabla 2. Comparación del porcentaje diario de germinación de semillas de ají para los tratamientos empleados. Promedios con letras distintas, en la misma columna, indican diferencia significativa según la prueba de Tukey $(P \leq 0,05)$.

\begin{tabular}{|c|c|c|c|c|c|c|}
\hline Día 7 & Día 8 & Día 9 & Día 10 & Día 11 & Día 12 & Día 13 \\
\hline $\begin{array}{c}\text { AC-60mT-120' } \\
3,25 \\
\text { A }\end{array}$ & $\begin{array}{c}\text { AC-60mT-120' } \\
13,50 \\
\text { A }\end{array}$ & $\begin{array}{c}\text { DC-60mT-120' } \\
30,50 \\
\text { A }\end{array}$ & $\begin{array}{c}\text { DC-60mT-120' } \\
47,50 \\
\text { A }\end{array}$ & $\begin{array}{c}\text { DC-60mT-120' } \\
63,00 \\
\text { A }\end{array}$ & $\begin{array}{c}\text { DC-60mT-120' } \\
73,00 \\
\text { A }\end{array}$ & $\begin{array}{c}\text { AC-60mT-60' } \\
78,75 \\
\text { A }\end{array}$ \\
\hline $\begin{array}{c}\text { DC-60mT-120' } \\
2,25 \\
\text { AB }\end{array}$ & $\begin{array}{c}\text { DC-60mT-120' } \\
12,50 \\
A B\end{array}$ & $\begin{array}{c}\text { AC-60mT-120' } \\
29,75 \\
\text { A }\end{array}$ & $\begin{array}{c}\text { AC-60mT-120' } \\
47,25 \\
\text { AB }\end{array}$ & $\begin{array}{c}\text { AC-60mT-60' } \\
59,75 \\
\text { A }\end{array}$ & $\begin{array}{c}\text { AC-60mT-60' } \\
71,25 \\
\text { AB }\end{array}$ & $\begin{array}{c}\text { AC-60mT-120 } \\
78,75 \\
\text { A }\end{array}$ \\
\hline $\begin{array}{c}\text { AC-60mT-60' } \\
1,75 \\
\text { AB }\end{array}$ & $\begin{array}{c}\text { DC-30mT-120' } \\
8,75 \\
\text { BC }\end{array}$ & $\begin{array}{c}\text { AC-60mT-60' } \\
25,00 \\
\text { AB }\end{array}$ & $\begin{array}{c}\text { AC-60mT-60' } \\
43,50 \\
\text { AB }\end{array}$ & $\begin{array}{c}\text { AC-60mT-120' } \\
59,00 \\
\text { A }\end{array}$ & $\begin{array}{c}\text { AC-60mT-120' } \\
70,50 \\
\text { AB }\end{array}$ & $\begin{array}{c}\text { AC-30mT-60' } \\
77,50 \\
\text { A }\end{array}$ \\
\hline $\begin{array}{c}\text { DC-30mT-120' } \\
1,50 \\
A B\end{array}$ & $\begin{array}{c}\text { AC-60mT-60' } \\
8,75 \\
\text { BC }\end{array}$ & $\begin{array}{c}\text { DC-30mT-120' } \\
22,50 \\
\text { AB }\end{array}$ & $\begin{array}{c}\text { DC-30mT-120' } \\
36,00 \\
\text { ABC }\end{array}$ & $\begin{array}{c}\text { AC-30mT-60' } \\
53,00 \\
\text { AB }\end{array}$ & $\begin{array}{c}\text { AC-30mT-60' } \\
70,50 \\
A B\end{array}$ & $\begin{array}{c}\text { DC-60mT-120 } \\
76,75 \\
\text { A }\end{array}$ \\
\hline $\begin{array}{c}\text { AC-30mT-120' } \\
1,50 \\
\text { A B }\end{array}$ & $\begin{array}{c}\text { AC-30mT-60' } \\
8,00 \\
\text { C } \\
\end{array}$ & $\begin{array}{c}\text { AC-30mT-60' } \\
19,50 \\
\text { BC } \\
\end{array}$ & $\begin{array}{c}\text { AC-30mT-60' } \\
33,00 \\
\text { ABC } \\
\end{array}$ & $\begin{array}{c}\text { DC-60mT-60' } \\
47,00 \\
\text { ABC }\end{array}$ & $\begin{array}{c}\text { DC-30mT-120' } \\
62,25 \\
\text { ABC }\end{array}$ & $\begin{array}{c}\text { DC-30mT-120 } \\
72,75 \\
\text { A }\end{array}$ \\
\hline $\begin{array}{c}\text { DC-60mT-60' } \\
1,00 \\
\text { AB }\end{array}$ & $\begin{array}{c}\text { AC-30mT-120' } \\
6,75 \\
\text { CD }\end{array}$ & $\begin{array}{c}\text { DC-60mT-60' } \\
18,75 \\
\text { BC }\end{array}$ & $\begin{array}{c}\text { DC-60mT-60' } \\
32,75 \\
\text { ABC }\end{array}$ & $\begin{array}{c}\text { AC-30mT-120' } \\
46,00 \\
\text { ABC }\end{array}$ & $\begin{array}{c}\text { DC-60mT-60' } \\
62,00 \\
\text { ABC }\end{array}$ & $\begin{array}{c}\text { DC-60mT-60' } \\
70,75 \\
\text { A }\end{array}$ \\
\hline $\begin{array}{c}\text { DC-30mT-60' } \\
0,75 \\
\text { AB }\end{array}$ & $\begin{array}{c}\text { DC-60mT-60' } \\
5,25 \\
\text { CDE }\end{array}$ & $\begin{array}{c}\text { AC-30mT-120' } \\
18,50 \\
\text { BC }\end{array}$ & $\begin{array}{c}\text { AC-30mT-120' } \\
31,50 \\
\text { BCD }\end{array}$ & $\begin{array}{c}\text { DC-30mT-120' } \\
45,00 \\
\text { ABC }\end{array}$ & $\begin{array}{c}\text { AC-30mT-120' } \\
61,75 \\
A B C\end{array}$ & $\begin{array}{c}\text { AC-30mT-120 } \\
68,50 \\
\text { A }\end{array}$ \\
\hline $\begin{array}{c}\text { AC-30mT-60' } \\
0,75 \\
A B\end{array}$ & $\begin{array}{c}\text { DC-30mT-60' } \\
3,25 \\
\text { DEF }\end{array}$ & $\begin{array}{c}\text { DC-30mT-60' } \\
10,75 \\
\text { CD }\end{array}$ & $\begin{array}{c}\text { DC-30mT-60' } \\
23,00 \\
\text { CDE }\end{array}$ & $\begin{array}{c}\text { DC-30mT-60' } \\
33,50 \\
\text { BCD }\end{array}$ & $\begin{array}{c}\text { AC-30mT-30' } \\
54,25 \\
\text { ABC }\end{array}$ & $\begin{array}{c}\text { AC-30mT-30' } \\
67,75 \\
\text { A }\end{array}$ \\
\hline $\begin{array}{c}\text { AC-30mT-30' } \\
0,50 \\
\text { B }\end{array}$ & $\begin{array}{c}\text { AC-30mT-30' } \\
1,25 \\
E F\end{array}$ & $\begin{array}{c}\text { TESTIGO } \\
5,25 \\
D\end{array}$ & $\begin{array}{c}\text { TESTIGO } \\
15,75 \\
\text { DE }\end{array}$ & $\begin{array}{c}\text { AC-30mT-30' } \\
31,25 \\
\text { CD }\end{array}$ & $\begin{array}{c}\text { DC-30mT-60' } \\
49,75 \\
\text { BC }\end{array}$ & $\begin{array}{c}\text { AC-60mT-30' } \\
61,50 \\
\text { A }\end{array}$ \\
\hline $\begin{array}{c}\text { Testigo } \\
0,00 \\
\text { B }\end{array}$ & $\begin{array}{c}\text { Testigo } \\
1,00 \\
\text { E F }\end{array}$ & $\begin{array}{c}\text { AC-30mT-30' } \\
4,50 \\
\text { D }\end{array}$ & $\begin{array}{c}\text { AC-30mT-30' } \\
15,25 \\
E\end{array}$ & $\begin{array}{c}\text { Testigo } \\
28,25 \\
\text { CD }\end{array}$ & $\begin{array}{c}\text { DC-30mT-30' } \\
46,25 \\
\text { C }\end{array}$ & $\begin{array}{c}\text { DC-30mT-60' } \\
61,25 \\
\text { A }\end{array}$ \\
\hline $\begin{array}{c}\text { DC-60mT-30' } \\
0,00 \\
\text { B }\end{array}$ & $\begin{array}{c}\text { AC-60mT-30' } \\
0,50 \\
F\end{array}$ & $\begin{array}{c}\text { DC-30mT-30' } \\
3,25 \\
\text { D }\end{array}$ & $\begin{array}{c}\text { DC-30mT-30' } \\
11,25 \\
E\end{array}$ & $\begin{array}{c}\text { DC-60mT-30' } \\
23,50 \\
\text { D }\end{array}$ & $\begin{array}{c}\text { Testigo } \\
43,75 \\
\text { C }\end{array}$ & $\begin{array}{c}\text { DC-60mT-30' } \\
60,50 \\
\text { A }\end{array}$ \\
\hline $\begin{array}{c}\text { DC-30mT-30' } \\
0,00 \\
\text { B }\end{array}$ & $\begin{array}{c}\text { DC-30mT-30' } \\
0,25 \\
F\end{array}$ & $\begin{array}{c}\text { DC-60mT-30' } \\
2,50 \\
\text { D }\end{array}$ & $\begin{array}{c}\text { DC-60mT-30' } \\
10,50 \\
\text { E }\end{array}$ & $\begin{array}{c}\text { DC-30mT-30' } \\
23,50 \\
\text { D }\end{array}$ & $\begin{array}{c}\text { AC-60mT-30' } \\
43,75 \\
\text { C }\end{array}$ & $\begin{array}{c}\text { DC-30mT-30' } \\
60,50 \\
\text { A }\end{array}$ \\
\hline $\begin{array}{c}\text { AC-60mT-30' } \\
0,00 \\
\text { B }\end{array}$ & $\begin{array}{c}\text { DC-60mT-30' } \\
0,00 \\
F\end{array}$ & $\begin{array}{c}\text { AC-60mT-30' } \\
2,50 \\
\text { D }\end{array}$ & $\begin{array}{c}\text { AC-60mT-30' } \\
7,75 \\
\text { E }\end{array}$ & $\begin{array}{c}\text { AC-60mT-30' } \\
20,00 \\
\text { D }\end{array}$ & $\begin{array}{c}\text { DC-60mT-30' } \\
42,75 \\
\text { C }\end{array}$ & $\begin{array}{c}\text { Testigo } \\
60,00 \\
\text { A }\end{array}$ \\
\hline
\end{tabular}

ción del agua debido principalmente a variación en la permeabilidad de la membrana celular (Osipova, 1990; Pietruszewski, 2011), transformaciones bioquímicas y orientación de las semillas con respecto a los campos magnéticos en donde su aplicación en sistemas biológicos puede explicarse mediante la transferencia de energía o electrones sobre la materia con contenido de radicales 
libres, que son atraídos o repelidos en función de su carga (Galland y Pazur, 2005). Al incrementarse la carga de dichos radicales se crea una bioestimulación dentro de la semilla, que permite acelerar ciertos procesos como son: biosíntesis de proteínas, actividad enzimática y la movilización de algunas sustancias de reserva hacia el eje embrionario, hecho que da lugar a la ruptura del endospermo y posteriormente a la emergencia de la radícula. Sin embargo, para que los procesos descritos anteriormente inicien dentro de la semilla, la respiración debe activarse, para así proporcionar el aporte de energía en forma de ATP y nutrientes para el crecimiento del embrión. Las semillas ricas en proteínas, como las de ají reaccionan más favorablemente a la aplicación de campos magnéticos que aquellas ricas en almidón y lípidos (Flórez et al., 2012). Rochalska y Orzeszko-Rywka (2008) afirman que los embriones que han tenido un mejor suministro de componentes orgánicos como lípidos, carbohidratos, proteínas, entre otros, presentan un desarrollo más rápido y adecuado de las plántulas, lo cual puede indicar que la activación temprana de la respiración de las semillas incrementa el vigor de las plantas, y posiblemente genera mejores respuestas fisiológicas durante todo el ciclo vegetativo.

A los 19 dds cuando se detuvo el proceso de germinación, los porcentajes de la misma estuvieron entre 84 y 91\%, correspondientes a los tratamientos testigo y AC-30mT-60' respectivamente. Los campos electromagnéticos implementados no tuvieron efecto en el porcentaje de germinación final de semillas de ají, Limo Rojo, ya que no se presentaron diferencias significativas entre tratamientos $(P>0,05)$. Los resultados indicaron que la estimulación electromagnética más que aumentar el porcentaje de germinación, aceleró dicho proceso, estos resultados pueden deberse a que los sistemas biológicos no son lineales (Méndez, 2005), por tanto no se pueden esperar los mismos resultados para todas las especies con las mismas características del campo aplicado (Cuero, 2004), ni tampoco la misma respuesta de una especie a la aplicación de diferentes campos electromagnéticos (Domínguez et al., 2010).

La aplicación de esta tecnología podría brindar grandes beneficios a los productores de plántulas de hortalizas y frutales, pues permite reducir los tiempos de germinación, logrando obtener mayor número de plántulas en menor tiempo, lo que se traduce en mayores ingresos.

\section{CONCLUSIONES}

La estimulación electromagnética de las semillas de ají tiene efecto en la reducción del periodo de germinación de las plántulas, cuando se incrementa tanto el tiempo de estimulación de las semillas como la intensidad del campo electromagnético aplicado. Sin embargo, el porcentaje de germinación final en semillas de ají estimuladas electromagnéticamente no presentó diferencia significativa con el porcentaje obtenido de las semillas no estimuladas. De acuerdo con los resultados es recomendable continuar estudiando el efecto de campos electromagnéticos con intensidades mayores a $60 \mathrm{mT}$, para determinar su efecto en el porcentaje de germinación total en la variedad de ají Limo Rojo.

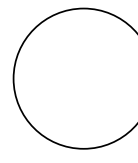

AGRADECIMIENTOS

Este trabajo fue financiado por la Universidad del Valle; Cristian Jiménez fue apoyado con el programa de joven investigador por parte del Instituto Colombiano de Ciencia y Tecnología, Colciencias. 


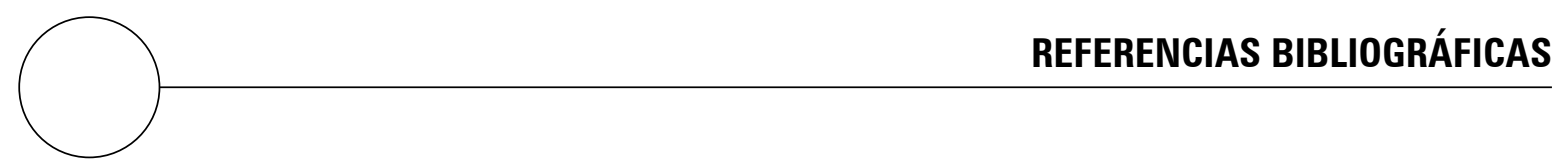

Biomagnetics USA. 2013. The revolutionary discoveries of Davis and Rawls. En: We have the science, http://www.magnetage.com; consulta: marzo de 2013.

Cuero, R. y R. Tulande. 2004. Evaluación de la estimulación magnética con campo magnético variable en la cachaza seca y en el periodo de compostaje de la cachaza verde de caña de azúcar (Saccharum officinarum L.) tratadas con microorganismos benéficos. Trabajo de grado. Facultad de Ingeniería, Universidad Nacional de Colombia, Palmira.

De Souza Torres, A., E. Porras y R. Casate. 1999. Efecto del tratamiento magnético de semillas de tomate (Lycopersicon esculentum Mill.) sobre la germinación y el crecimiento de las plántulas. Invest. Agr Prod. Prot. Veg. 14(3), 437-444.

Domínguez, P.A., A.C. Hernández, O.A. Cruz, A. Carballo, B.R. Zepeda y O.E. Martínez. 2010. Semilla de maíz bajo la influencia de irradiación de campos electromagnéticos. Rev. Fitotec. Mex. 33(2), 183188.

Flórez, M., M.V. Carbonell y E. Martínez. 2007. Exposure of maize seeds to stationary magnetic fields: Effects on germination and early growth. Environ. Exp. Bot. 59(1), 68-75.

Flórez, M., E. Martínez y M.V. Carbonell. 2012. Effect of magnetic field treatment on germination of medicinal plants Salvia officinalis L. and Calendula officinalis L. Pol. J. Environ. Stud. 21, 57-63.

Galland, P. y A. Pazur. 2005. Magnetoreception in plants. J. Plant Res. 118, 371-89.

Marks, N. y P.S. Szecówka. 2010. Impact of variable magnetic field stimulation on growth of aboveground parts of potato plants. Inter. Agrophy. 24, 165-170.

Méndez, R. 2005. La evolución biológica como sistema autoorganizado, dinámicas no lineales y sistemas biológicos. Ciencias 79, 26-34.
Ministerio de Agricultura y Desarrollo Rural. 2012. Anuario estadístico de frutas y hortalizas 2007 2011 y sus calendarios de siembras y cosechas. JL Impresores Ltda., Bogotá. pp. 189-191.

Osipova, L.D. 1990. Influencia de los campos magnéticos sobre los tejidos de callos de frutales (en ruso). Bull. Cent. Ord. Trud. Krasn. Zn. Genet. Lab. Im. Michurina 49, 22-25.

Pietruszewski, S. 2011. Electromagnetic fields, impact on seed. Germination and plant growth. pp. $267-$ 269. En: Glinski J., J. Horabik y J. Lipiec (eds.). Encyclopedia of agrophysics. Earth and Environmental Science. Editorial Board, Lublin, Polonia.

Racuciu, M., D. Creanga e I. Horga. 2008. Plant growth under static magnetic field influence. Rom. J. Phys. 53(1-2), 353-359.

Revista Cambio. 2008. Entrevista al gerente de la empresa Hugo Restrepo y Cia., 13 de febrero de 2008. En: http://www.eltiempo.com/archivo/documento/ CMS-3957397; consulta: abril de 2011.

Rochalska, M. y K. Grabowska. 2007. Influence of magnetic fields on activity of enzyme: $a-$ and $b$ -amylase and glutathione S-transferase (GST) in wheat plants. Inter. Agrophy. 21, 185-188.

Rochalska, M. y A. Orzeszko-Rywka. 2008. Influence of alternating magnetic field on respiration of sugar beet seeds. Inter. Agrophy. 22, 255-259.

Torres, C., J. Díaz y P. Cabal. 2008. Efecto de campos magnéticos en la germinación de semillas de arroz (Oryza sativa L.) y tomate (Solanum lycopersicum L.). Agron. Colomb. 26(2), 177-185.

Vashisth, A. y S. Nagarajan. 2010. Effect on germination and early growth characteristics in sunflower $(\mathrm{He}$ lianthus annuus) seeds exposed to static magnetic field. J. Plant Physiol. 167, 149-156.

Vásquez, V., M. Gómez, B. Segura y Rosales-Rivera. 2006. Efectos de campos magnéticos en material orgánico. Rev. Col. Fís. 38(3), 1307-1310. 